Appl. Set-Valued Anal. Optim. 1 (2019), No. 3, pp. 319-335

Available online at http://asvao.biemdas.com

https://doi.org/10.23952/asvao.1.2019.3.06

\title{
DUALITY IN VECTOR OPTIMIZATION WITH DOMINATION STRUCTURES
}

\author{
TRUONG QUANG BAO ${ }^{1}$, THANH TAM LE ${ }^{2,3}$, CHRISTIANE TAMMER $^{2, *}$, VU ANH TUAN ${ }^{2}$ \\ ${ }^{1}$ Department of Mathematics and Computer Science, Northern Michigan University \\ ${ }^{2}$ Institute of Mathematics, Faculty of Natural Sciences II, Martin-Luther-University Halle-Wittenberg \\ ${ }^{3}$ Faculty of Basic Sciences, University of Transport and Communications, Hanoi, Vietnam
}

\begin{abstract}
In this paper, we study duality schemes for vector optimization problems with respect to variable domination structures by using an appropriate extension of the so-called Gerstewitz nonlinear scalarization function for dealing with weakly nondominated solutions. We apply these results to multiobjective location problems with variable domination structures. For nondominated solutions, we use a general scalarization function which is strictly monotone with respect to the domination structure. The strict monotonicity of our proposed function is also discussed.
\end{abstract}

Keywords. Vector optimization; Variable domination structure; Duality; Nonlinear scalarization function.

2010 Mathematics Subject Classification. 46A40, 46A20, 46B40, 49J53.

\section{INTRODUCTION AND NOTATIONS}

Recently, vector optimization problems (VOP) with domination structures have been intensively studied since they have many important applications, see $[6,7,11]$ and references therein. Vector optimization is a special case, where the domination structure is a constant map whose values are identical to a fixed ordering cone. Since domination sets vary from one element to another, we are able to define more than one binary relations, one of which defines one type of solutions. For an overview on solution concepts, characterizations of the solutions and numerical methods for solving VOP with variable ordering structures, see [6].

Duality or the duality principle has been an important topic in both scalar and vector optimization. A solution to the dual problem provides a lower bound to the solution of the primal minimization problem. The difference between the optimal values of a primal problem and its dual problem is called the duality gap. In general, it is not necessarily zero. In some situations, the formulation of a dual problem is simpler than its primal problem and thus it is easier to deal with the dual than to handle directly the primal problem. There are several approaches for duality in vector optimization; for example, conjugation, Lagrangian, axiomatic duality, etc.; see, $[3,9,12]$. An overview on concepts to dualization in vector optimization with fixed ordering structure is given in the book by Bot,, Grad and Wanka [3] (see also the references therein).

\footnotetext{
${ }^{*}$ Corresponding author.

E-mail addresses: btruong@nmu.edu (T.Q. Bao), le.thanh-tam@mathematik.uni-halle.de (T.T. Le), christiane. tammer@mathematik.uni-halle.de (C. Tammer), anh.vu@mathematik.uni-halle.de (V.A. Tuan).

Received November 15, 2019; Accepted December 17, 2019.
}

(C)2019 Applied Set-Valued Analysis and Optimization 
Our intention is to derive duality assertions for vector optimization problems with variable domination structures. In the book by Eichfelder [6] (see also [5]), duality assertions for vector optimization problems with a variable domination structure given by a cone-valued ordering map are derived. One approach, studied in [6, Section 8.2], is based on linear scalarization. Furthermore, an approach to duality for vector optimization problems with a variable domination structure defined by the Bishop-Phelps cone is derived in [6, Section 8.3] using a corresponding nonlinear scalarization.

The aim of our paper is to derive duality assertions for vector optimization problems with a variable domination structure given by a set-valued map. Based on a scalarization by means of a new nonlinear scalarization function (an extention of the so-called Gerstewitz function), we will use a Lagrangian approach in order to formulate the dual problem and to derive the weak and strong duality statements for weakly nondominated solutions to this general class of problems. Moreover, we show Lagrangian duality assertions for nondominated solutions using a scalarization function which is strictly monotone with respect to the domination structure.

The paper is organized as follows. In Section 2, we recall preliminaries from vector optimization with variable domination structures. Section 3 is devoted to properties of a nonlinear scalarization function associated with a domination structure. It plays an important role in constructing a useful Lagrange function in Section 4. We provide both weak duality and strong duality results for weakly nondominated solutions of a primal vector optimization problem with variable domination structure. In Section 4, we construct a Lagrange function associated to a set of strictly monotone scalarization functions to work with nondominated solutions of the primal problem. Section 5 ends this paper.

\section{PRELIMINERIES}

Throughout the paper, unless otherwise specified, $X$ and $Y$ are two normed vector spaces with their topological duals $X^{*}$ and $Y^{*}$, respectively. The norm in $X$ is denoted by $\|\cdot\|_{X}$. The ball centered at $x \in X$ and radius $\varepsilon>0$ is denoted by $\mathscr{B}(x, \varepsilon):=\left\{u \in X:\|x-u\|_{X} \leq \varepsilon\right\}$. $\overline{\mathbb{R}}:=\mathbb{R} \cup\{ \pm \infty\}$ and $\mathbb{R}_{+}$stand for the set of extended real numbers and the set of nonnegative real numbers.

Let $A \subseteq X$ be a nonempty set in $X$. The symbols int $A, \operatorname{cl} A$ and $\mathrm{bd} A$ stand for the interior, closure and boundary of $A$, respectively. $A$ is convex if for all $x, y \in A$ and for all $\lambda \in[0,1]$, one has $\lambda x+(1-\lambda) y \in A$. The cone generated by $A$ is defined by cone $A:=\{\lambda a: \lambda \geq 0$ and $a \in A\}$. The linear hull and the affine hull of $A$ are respectively defined by

$$
\begin{aligned}
\operatorname{lin}(A) & :=\left\{\sum_{i=1}^{n} \lambda_{i} x_{i}: n \in \mathbb{N}, x_{i} \in A, \lambda_{i} \in \mathbb{R}, i=1, \ldots, n\right\} \text { and } \\
\operatorname{aff}(A) & :=\left\{\sum_{i=1}^{n} \lambda_{i} x_{i}: n \in \mathbb{N}, x_{i} \in A, \lambda_{i} \in \mathbb{R}, i=1, \ldots, n, \sum_{i=1}^{n} \lambda_{i}=1\right\} .
\end{aligned}
$$

The strong quasi relative interior and the relative interior of $A$ are defined by

$$
\begin{aligned}
\operatorname{sqri}(A) & :=\{x \in A: \operatorname{cone}(A-x) \text { is a closed linear subspace }\} \text { and } \\
\operatorname{ri}(A) & :=\{x \in A: \exists \varepsilon>0, B(x, \varepsilon) \cap \operatorname{aff} A \subseteq A\} .
\end{aligned}
$$

In finite dimensional spaces, these two interior notions are identical. 
Let $Q$ be a nonempty set in $Y$. $Q$ is a cone if for every $q \in Q$ and for every $\lambda \geq 0$, one has $\lambda q \in Q$. A cone $Q$ is convex if and only if $Q+Q=Q$. Furthermore, $Q$ is pointed if $Q \cap(-Q)=$ $\{0\}$ and $Q$ is proper if $Q \neq\{0\}$ and $Q \neq Y$. The positive orthant $\mathbb{R}_{+}^{n}:=\left\{\left(x_{1}, \ldots, x_{n}\right) \in \mathbb{R}^{n}: \forall i \in\right.$ $\left.\{1, \ldots, n\}, x_{i} \geq 0\right\}$ is a closed, convex, pointed and proper cone of $\mathbb{R}^{n}$.

Let $\varphi: X \rightarrow \overline{\mathbb{R}}$ be a function. Its domain and epigraph are respectively given by

$$
\operatorname{dom} \varphi:=\{x \in X: f(x)<+\infty\} \text { and epi } \varphi:=\{(x, t) \in Z \times \mathbb{R}: f(x) \leq t\} .
$$

$\varphi$ is proper if $\operatorname{dom} \varphi \neq \emptyset$ and for all $x \in X$, one has $\varphi(x) \neq-\infty$. $\varphi$ is convex if epi $\varphi$ is a convex set.

A set-valued map $\mathscr{K}: Y \rightrightarrows Y$ is called a domination structure of the space $Y$ with dom $\mathscr{K} \neq \emptyset$, where $\operatorname{dom} \mathscr{K}:=\{y \in Y: \mathscr{K}(y) \neq \emptyset\}$. In this paper, we will use the following properties of the domination structure $\mathscr{K}$ :

(C1) $\forall y \in Y, \mathscr{K}(y)$ is a closed, pointed and proper set.

(C2) $\exists k \in \bigcap_{y \in Y}$ int $\mathscr{K}(y)$ and $\forall y \in Y, \mathscr{K}(y)+(0,+\infty) k \subseteq$ int $\mathscr{K}(y)$.

(C2)' $\exists k \in \bigcap_{y \in Y} \mathscr{K}(y)$ and $\forall y \in Y, \mathscr{K}(y)+[0,+\infty) k \subseteq \mathscr{K}(y)$.

(C3) $\forall y \in Y, \exists t \in \mathbb{R}, t k \notin y+\mathscr{K}(y)-\xi$, where $\xi$ is specified later and $k$ is given by (C2) or (C2)'.

(C4) $\mathscr{K}$ is convex on a convex set $\Xi$ in the sense that

$$
\forall \lambda \in[0,1], \forall y_{1}, y_{2} \in \Xi, \quad \lambda \mathscr{K}\left(y_{1}\right)+(1-\lambda) \mathscr{K}\left(y_{2}\right) \subseteq \mathscr{K}\left(\lambda y_{1}+(1-\lambda) y_{2}\right),
$$

where $\Xi$ will be specified later.

Obviously, if $\mathscr{K}(y) \equiv Q$ for all $y \in Y$, where $Q$ is a closed, convex, pointed and proper cone of $Y$, conditions (C1), (C2)' and (C4) are automatically satisfied. Additionally, if int $Q \neq \emptyset$ then conditions (C2) and (C3) are fulfilled as well.

Given a domination structure $\mathscr{K}$ of the space $Y$. For every $y_{1}, y_{2} \in Y$, we study the following binary relation $\preceq \mathscr{K}$ defined by

$$
y_{1} \preceq \mathscr{K} y_{2} \quad: \Longleftrightarrow y_{2} \in y_{1}+\mathscr{K}\left(y_{1}\right) .
$$

The weak relation of $\preceq \mathscr{K}$ is defined by replacing the domination set in (2.1) with its interior; i.e.,

$$
y_{1} \prec \mathscr{K} y_{2} \quad: \Longleftrightarrow \quad y_{2} \in y_{1}+\text { int } \mathscr{K}\left(y_{1}\right) \text {. }
$$

Example 2.1. Take an element $k=(1,1) \in \mathbb{R}^{2}$ and a positive real number $0<\varepsilon<1$. Consider a domination structure $\mathscr{K}: \mathbb{R}^{2} \rightrightarrows \mathbb{R}^{2}$ given by

$$
\mathscr{K}(x, y):=\left[0,|x|^{1 / 4}+\varepsilon\right] \times\left[0,|y|^{1 / 4}+\varepsilon\right]+\mathbb{R}_{+} k .
$$

It is easy to check that $\mathscr{K}$ satisfies conditions (C1), (C2) and (C2'). In addition, for all $x_{1}, x_{2} \in$ $\mathbb{R}_{+}$and $\alpha \in(0,1)$ it holds that

$$
\alpha x_{1}^{\frac{1}{4}}+(1-\alpha) x_{2}^{\frac{1}{4}} \leq\left(\alpha x_{1}+(1-\alpha) x_{2}\right)^{\frac{1}{4}} .
$$

Therefore, $\mathscr{K}$ is convex on $\mathbb{R}_{+}^{2}$. However, the aforementioned inequality does not hold for all $x_{1}, x_{2} \in \mathbb{R}$ and thus $\mathscr{K}$ is not convex on $\mathbb{R}^{2}$. For an illustration of this domination structure, see Figure 1.

Next, we recall the concept of nondominatedness to sets. 


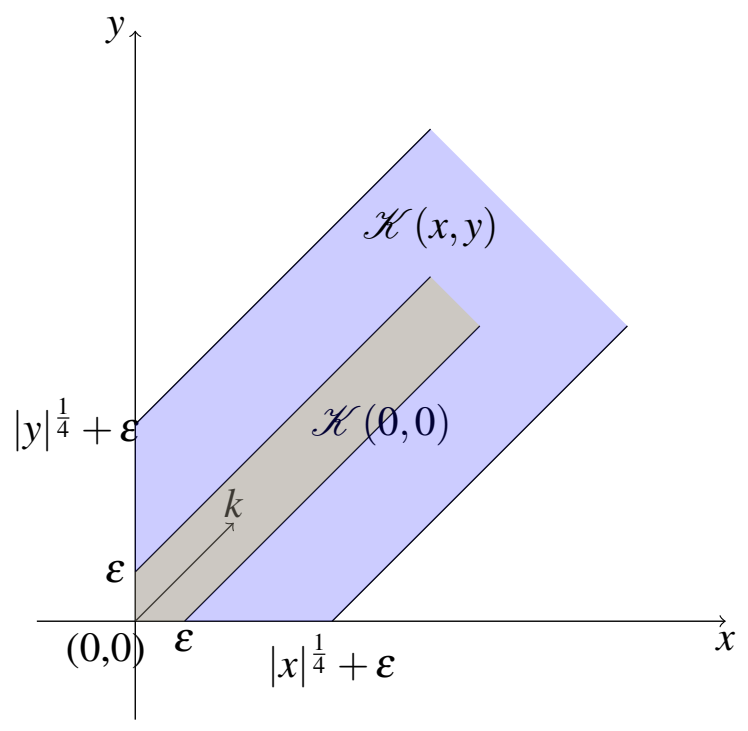

FIGURE 1. Images of $\mathscr{K}$ in Example 2.1 are depicted at $(0,0)$ and $(x, y)$.

Definition 2.1. Let $A \subseteq Y$ be a nonempty subset, $\bar{y} \in A$ and $\mathscr{K}: Y \rightrightarrows Y$ be a domination structure of $Y$ satisfying $(\mathrm{C} 1)$. We say that

(i) $\bar{y}$ is a $\preceq \mathscr{K}$-minimal point or a nondominated point of $A$ with respect to $\mathscr{K}$ if for all $y \in$ $A \backslash\{\bar{y}\}$, one has $y \npreceq \mathscr{K} \bar{y}$.

(ii) $\bar{y}$ is a weakly nondominated point of $A$ with respect to $\mathscr{K}$ if for all $y \in A$, one has $y \nprec \mathscr{K} \bar{y}$.

(iii) $\bar{y}$ is a maximal point of $A$ with respect to $\mathscr{K}$ if for all $y \in A \backslash\{\bar{y}\}$, one has $\bar{y} \npreceq \mathscr{K} y$. Equivalently,

$$
\forall y \in A \backslash\{\bar{y}\}, y \notin \bar{y}+\mathscr{K}(\bar{y}) \Longleftrightarrow A \cap(\bar{y}+\mathscr{K}(\bar{y}))=\{\bar{y}\} .
$$

(iv) $\bar{y}$ is a weakly maximal point of $A$ with respect to $\mathscr{K}$ if for all $y \in A \backslash\{\bar{y}\}$, one has $\bar{y} \nprec \mathscr{K} y$. Equivalently,

$$
A \cap(\bar{y}+\operatorname{int} \mathscr{K}(\bar{y}))=\emptyset .
$$

The set of all nondominated points (respectively, weakly nondominated points, maximal points and weakly maximal points) of $A$ w.r.t. $\mathscr{K}$ are denoted by $\mathrm{ND}(A, \mathscr{K})$ (respectively, $\operatorname{WND}(A, \mathscr{K}), \operatorname{Max}(A, \mathscr{K})$ and $\operatorname{WMax}(A, \mathscr{K}))$.

Remark 2.1. 1. When $\mathscr{K}(y) \equiv Q$ for some closed, convex, and pointed cone $Q \subset Y$, both nondominated and maximal points of $A$ reduce to the classical Pareto minimal and maximal points of $A$ with respect to the ordering cone $Q$. In this case, $\operatorname{ND}(A, \mathscr{K})=\operatorname{Min}(A, Q)$, $\operatorname{WND}(A, \mathscr{K})=\mathrm{WMin}(A, Q), \operatorname{Max}(A, \mathscr{K})=\operatorname{Max}(A, Q)$ and $\operatorname{WMax}(A, \mathscr{K})=\operatorname{WMax}(A, \mathscr{K})$.

2. $\bar{y} \in \operatorname{Max}(A, \mathscr{K})$ is equivalent to $\bar{y} \in \operatorname{Max}(A, \mathscr{K}(\bar{y}))$.

3. In vector optimization with domination structure, there is another binary relation which is denoted by $\preceq_{\mathscr{K}}^{2}$ and uses the domination set of the bigger point in the ordering formulation; i.e.,

$$
y_{1} \preceq_{\mathscr{K}}^{2} y_{2}: \Longleftrightarrow y_{1} \in y_{2}-\mathscr{K}\left(y_{2}\right) .
$$

Therefore, if $\bar{y}$ is a $\preceq_{\mathscr{K}}^{2}$-minimal point of $A$, then it is a nondominated point of $A$ with respect to the constant domination structure $\mathscr{C}(y) \equiv \mathscr{K}(\bar{y})$ for all $y \in Y$. If $\bar{y}$ is a $\preceq_{\mathscr{K}}^{2}$-maximal point of $A$, 
then it is a nondominated point of $A$ with respect to the domination structure $\mathscr{D}(y):=-\mathscr{K}(y)$ for all $y \in Y$.

Finally, we extend the convexity property of vector-valued functions from fixed ordering cones to domination structures.

Definition 2.2. Let $f: X \rightarrow Y$ be a vector valued function and $\mathscr{K}: Y \rightrightarrows Y$ be a domination structure on $Y$. We say that $f$ is $\mathscr{K}$-convex on a convex set $\Xi \subseteq X$ if its epigraphical map is convex on $\Xi \subseteq X$, where the epigraphical map of $f$ with respect to $\mathscr{K}$ is defined by

$$
\mathscr{E}_{f}(x):= \begin{cases}f(x)+\mathscr{K}(f(x)) & \text { if } x \in \operatorname{dom} f \\ \emptyset & \text { otherwise. }\end{cases}
$$

Observe that Definition 2.2 reduces to the notion of cone-convexity of vector-valued functions when $\mathscr{K}(y) \equiv Q$ for some pointed convex cone $Q \subseteq Y$, see [13]. Observe also that $f$ is $\mathscr{K}$ convex if and only if its epigraph is convex. This type of convexity is different from other kinds in $[2,4]$.

Example 2.2. Let $k, \varepsilon$ and the domination structure $\mathscr{K}: \mathbb{R}^{2} \rightrightarrows \mathbb{R}^{2}$ as in Example 2.1. Consider a function $f: \mathbb{R} \rightarrow \mathbb{R}^{2}$ given by:

$$
\forall x \in \mathbb{R}: f(x)=\left(x^{2}, x^{2}\right)
$$

We have

$$
\mathscr{K}(f(x))=\left[0,|x|^{1 / 2}+\varepsilon\right] \times\left[0,|x|^{1 / 2}+\varepsilon\right]+\mathbb{R}_{+} k .
$$

For every $x, y \in \mathbb{R}_{+}$and $\alpha \in(0,1)$, it holds that

$$
\alpha f(x)+(1-\alpha) f(y) \in f(\alpha x+(1-\alpha) y)+\mathbb{R}_{+} k
$$

and

$$
\alpha \mathscr{K}(f(x))+(1-\alpha) \mathscr{K}(f(y)) \subseteq \mathscr{K}(f(\alpha x+(1-\alpha) y)) .
$$

These inclusions verify the $\mathscr{K}$-convexity of $f$ over $\mathbb{R}_{+}$. For an illustration, see Figure 2 where the blue part is the image of $\mathscr{K}(f(\alpha x+(1-\alpha) y))$ and $\alpha \mathscr{K}(f(x))+(1-\alpha) \mathscr{K}(f(y))$ is depicted by the red one.

However, $f$ is not $\mathscr{K}$-convex on $\mathbb{R}$. Indeed, take $x=1, y=-1, \alpha=\frac{1}{2}$. Then, $f(x)=f(y)=$ $(1,1), \mathscr{K}(f(x))=\mathscr{K}(f(y))=[0,1+\varepsilon] \times[0,1+\varepsilon]+\mathbb{R}_{+} k$. On the other hand,

$$
f(\alpha x+(1-\alpha) y)=f(0)=(0,0)
$$

and

$$
\mathscr{K}(f(0))=[0, \varepsilon] \times[0, \varepsilon]+\mathbb{R}_{+} k
$$

Therefore,

$$
\frac{1}{2}(f(1)+\mathscr{K}(f(1)))+\frac{1}{2}(f(-1)+\mathscr{K}(f(-1))) \nsubseteq \subseteq f(0)+\mathscr{K}(f(0)) .
$$




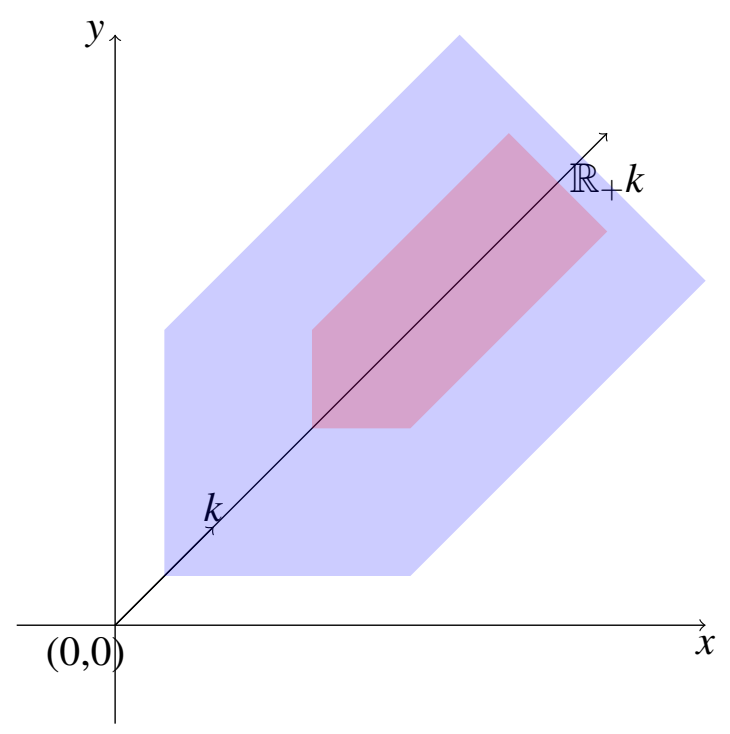

FIGURE 2. Illustration of Example 2.2

\section{A SCALARIZATION METHOD IN VECTOR OPTIMIZATION W.R.T. VARIABLE DOMINATION STRUCTURES}

In this section, we study a scalarization function which is used to construct the dual problem of a primal vector optimization problem with respect to a domination structure and to investigate both weak and strong duality theorems for this pair of problems. This scalarization was studied in [7, Section 5.2.1] and can be viewed as an extension of the Gerstewitz nonlinear scalarization function; see, e.g., [8], from a domination set to a convex-cone-valued domination structure; known also as variable ordering structure. Here, we further extend it to general domination structures. Precisely, we study some important properties of the function when domination sets are not convex cones.

Throughout this section, we assume that the pair $\{\mathscr{K}, k\}$ satisfies conditions (C1) and (C2). For a given element $\xi \in Y$, we consider the scalarization function $T_{\xi}: Y \rightarrow \overline{\mathbb{R}}$ defined by

$$
T_{\xi}(y):=\inf \{t \in \mathbb{R}: t k \in y+\mathscr{K}(y)-\xi\} \text { with } \inf \emptyset:=+\infty .
$$

Obviously, $\operatorname{dom} T_{\xi}=\{y \in Y: \mathbb{R} k \cap(y+\mathscr{K}(y)-\xi) \neq \emptyset\}$. By $(\mathrm{C} 1), T_{\xi}(\xi)=0$. This function is used in [6, Section 5.2.1] to characterize nondominated elements of a set with variable ordering maps $\mathscr{K}$; i.e., $\mathscr{K}(y)$ is a closed, pointed, convex and proper cone for all $y \in Y$. It is also important to mention that the condition $(\mathrm{C} 1)$ is needed in order to derive duality assertions, see also [6, Chapter 8].

Definition 3.1. A function $s: Y \rightarrow \mathbb{R}$ is said to be $\mathscr{K}$-monotone if

$$
\forall y_{1}, y_{2} \in Y, y_{1} \preceq \mathscr{K} y_{2} \Longrightarrow s\left(y_{1}\right) \leq s\left(y_{2}\right) .
$$

It is called strictly $\mathscr{K}$-monotone if

$$
\forall y_{1}, y_{2} \in Y, y_{1} \preceq \mathscr{K} y_{2} \text { and } y_{1} \neq y_{2} \Longrightarrow s\left(y_{1}\right)<s\left(y_{2}\right) .
$$

The next theorem provides important properties of the scalarization function $T_{\xi}$. 
Theorem 3.1. Suppose that $\mathscr{K}: Y \rightrightarrows Y$ satisfies $(C 1)$ and $(C 2)$ and $\xi \in Y$. The scalarization function $T_{\xi}$ defined by (3.1) has the following properties:

(i) If $T_{\xi}(y)$ is finite for some $y \in Y$, then

$$
T_{\xi}(y)=\min \{t \in \mathbb{R}: t k \in y+\mathscr{K}(y)-\xi\} .
$$

(ii) Assume $T_{\xi}(y)$ is finite. Then $\forall t \geq 0,\left(T_{\xi}(y)+t\right) k \in y+\mathscr{K}(y)-\xi$.

(iii) Assume $T_{\xi}(y)=-\infty$. Then, $\forall t \in \mathbb{R}, t k \in y+\mathscr{K}(y)-\xi$.

(iv) $T_{\xi}$ is proper if and only if (C3) holds.

(v) If (C4) holds with $\Xi=\operatorname{dom} T_{\xi}$, then $T_{\xi}$ is convex.

(vi) If $f$ is $\mathscr{K}$-convex on $\Xi$, where $\Xi \subseteq X$ is convex, then the composition $h:=T_{\xi} \circ f$ is convex on $\Xi$.

(vii) If $\mathscr{K}(y)+\mathscr{K}(y+d) \subseteq \mathscr{K}(y)$ for all $d \in \mathscr{K}(y)$ then $T$ is $\mathscr{K}$-monotone.

(viii) If (C2') holds, then:

$$
T_{\xi}(y)<\lambda \quad \Longleftrightarrow \quad \lambda k \in y+i n t \mathscr{K}(y)+\xi .
$$

Proof. (i) Assume that $T_{\xi}(y)$ is finite. Set

$$
t_{0}:=T_{\xi}(y)=\inf \{t \in \mathbb{R}: t k \in y+\mathscr{K}(y)-\xi\} \in \mathbb{R}
$$

By (C2), we have

$$
\forall t>t_{0}, t k \in y+\mathscr{K}(y)-\xi .
$$

By $(\mathrm{C} 1), t_{0} k \in y+\mathscr{K}(y)-\xi$ clearly verifying

$$
t_{0}=\min \{t \in \mathbb{R}: t k \in y+\mathscr{K}(y)-\xi\} .
$$

(ii) Assume that $T_{\xi}(y)=t_{0} \in \mathbb{R}$. By (i), we have

$$
t_{0} k \in y+\mathscr{K}(y)-\xi .
$$

For every arbitrary number $t \geq 0$, we have

$$
\left(t_{0}+t\right) k \in y+(\mathscr{K}(y)+t k)-\xi \subseteq y+\mathscr{K}(y)-\xi,
$$

where the latter estimate holds due to (C2).

(iii) Assume that $T_{\xi}(y)=-\infty$. For every $t \in \mathbb{R}$, by the definition of $T_{\xi}$, we can find $\theta<t$ such that $\theta k \in y+\mathscr{K}(y)-\xi$. Taking into account (C2), we have

$$
t k \in y+\mathscr{K}(y)+(t-\theta) k-\xi \subseteq y+\mathscr{K}(y)-\xi .
$$

(iv) Fix an arbitrary element $y \in Y$ such that $T_{\xi}(y)=-\infty$. By (iii), we have

$$
T_{\xi}(y)=-\infty \Leftrightarrow \forall t \in \mathbb{R}, t k \in y+\mathscr{K}(y)-\xi \Leftrightarrow \xi-y+\mathbb{R} k \subseteq \mathscr{K}(y),
$$

i.e., the domination set $\mathscr{K}(y)$ contains a line parallel to the vector $k$. Therefore, $(i v)$ holds.

(v) Take $y_{1}, y_{2} \in \operatorname{dom} T_{\xi}$. Set

$$
t_{1}:=T_{\xi}\left(y_{1}\right) \text { and } t_{2}:=T_{\xi}\left(y_{2}\right) .
$$

By the definition of $T_{\xi}$ in (3.1), for every $\varepsilon>0$, we have

$$
\left(t_{1}+\varepsilon\right) k \in y_{1}+\mathscr{K}\left(y_{1}\right)-\xi \text { and }\left(t_{2}+\varepsilon\right) k \in y_{2}+\mathscr{K}\left(y_{2}\right)-\xi .
$$

Taking into account (C4), for every $\lambda \in(0,1)$ we have 


$$
\begin{aligned}
{\left[\lambda t_{1}+(1-\lambda) t_{2}+\varepsilon\right] k } & \in\left[\lambda y_{1}+(1-\lambda) y_{2}\right]+\left[\lambda \mathscr{K}\left(y_{1}\right)+(1-\lambda) \mathscr{K}\left(y_{2}\right)\right]-\xi \\
& \subseteq\left[\lambda y_{1}+(1-\lambda) y_{2}\right]+\mathscr{K}\left(\lambda y_{1}+(1-\lambda) y_{2}\right)-\xi .
\end{aligned}
$$

It follows that

$$
\begin{aligned}
T_{\xi}\left(\lambda y_{1}+(1-\lambda) y_{2}\right) & \leq \lambda t_{1}+(1-\lambda) t_{2}+\varepsilon \\
& =\lambda T_{\xi}\left(y_{1}\right)+(1-\lambda) T_{\xi}\left(y_{2}\right)+\varepsilon .
\end{aligned}
$$

Since $\varepsilon$ was arbitrary, we have

$$
T_{\xi}\left(\lambda y_{1}+(1-\lambda) y_{2}\right) \leq \lambda T_{\xi}\left(y_{1}\right)+(1-\lambda) T_{\xi}\left(y_{2}\right)
$$

clearly verifying the convexity of the scalarization function $T_{\xi}$.

(vi) For every $x_{1}, x_{2} \in \operatorname{dom} h$, we have

$$
t_{1}:=h\left(x_{1}\right)=T_{\xi}\left(f\left(x_{1}\right)\right) \text { and } t_{2}:=h\left(x_{2}\right)=T_{\xi}\left(f\left(x_{2}\right)\right) .
$$

By the definition of $T_{\xi}$ in (3.1), for every $\varepsilon>0$, we have

$$
\left(t_{1}+\varepsilon\right) k \in y_{1}+\mathscr{K}\left(y_{1}\right)-\xi \text { and }\left(t_{2}+\varepsilon\right) k \in y_{2}+\mathscr{K}\left(y_{2}\right)-\xi .
$$

Taking into account (C4), for every $\lambda \in(0,1)$ we have

$$
\begin{aligned}
{\left[\lambda t_{1}+(1-\lambda) t_{2}+\varepsilon\right] k } & \in\left[\lambda y_{1}+(1-\lambda) y_{2}\right]+\left[\lambda \mathscr{K}\left(y_{1}\right)+(1-\lambda) \mathscr{K}\left(y_{2}\right)\right]-\xi \\
& \subseteq\left[\lambda y_{1}+(1-\lambda) y_{2}\right]+\mathscr{K}\left(\lambda y_{1}+(1-\lambda) y_{2}\right)-\xi
\end{aligned}
$$

It follows that

$$
\begin{aligned}
T_{\xi}\left(\lambda y_{1}+(1-\lambda) y_{2}\right) & \leq \lambda t_{1}+(1-\lambda) t_{2}+\varepsilon \\
& =\lambda T_{\xi}\left(y_{1}\right)+(1-\lambda) T_{\xi}\left(y_{2}\right)+\varepsilon .
\end{aligned}
$$

Since $\varepsilon$ was arbitrary, we have

$$
T_{\xi}\left(\lambda y_{1}+(1-\lambda) y_{2}\right) \leq \lambda T_{\xi}\left(y_{1}\right)+(1-\lambda) T_{\xi}\left(y_{2}\right)
$$

clearly verifying the convexity of the scalarization function $T_{\xi}$.

Let $t_{1}, t_{2} \in \mathbb{R}$ such that

$$
t_{1} k \in f\left(x_{1}\right)+\mathscr{K}\left(f\left(x_{1}\right)\right)-\xi
$$

and

$$
t_{2} k \in f\left(x_{2}\right)+\mathscr{K}\left(f\left(x_{2}\right)\right)-\xi .
$$

Because of the $\mathscr{K}$-convexity of $f$ (see Definition 2.2), it follows that

$$
\begin{aligned}
{\left[\lambda t_{1}+(1-\lambda) t_{2}\right] k } & \in \lambda\left[f\left(x_{1}\right)+\mathscr{K}\left(f\left(x_{1}\right)\right)\right]+(1-\lambda)\left[f\left(x_{2}\right)+\mathscr{K}\left(f\left(x_{2}\right)\right)\right]-\xi \\
& \in f\left(\lambda x_{1}+(1-\lambda) x_{2}\right)+\mathscr{K}\left(f\left(\lambda x_{1}+(1-\lambda) x_{2}\right)\right)-\xi .
\end{aligned}
$$

It follows that

$$
h\left(\lambda x_{1}+(1-\lambda) x_{2}\right) \leq \lambda t_{1}+(1-\lambda) t_{2}
$$


Thus,

$$
h\left(\lambda x_{1}+(1-\lambda) x_{2}\right) \leq \lambda h\left(x_{1}\right)+(1-\lambda) h\left(x_{2}\right) .
$$

(vii) Let $y_{1}, y_{2} \in Y$ such that $y_{1} \prec \mathscr{K} y_{2}$; i.e., $y_{2} \in y_{1}+\mathscr{K}\left(y_{1}\right)$. From the additional assumption of $\mathscr{K}$, we have that

$$
y_{2}+\mathscr{K}\left(y_{2}\right) \subseteq y_{1}+\mathscr{K}\left(y_{1}\right)+\mathscr{K}\left(y_{1}+\mathscr{K}\left(y_{1}\right)\right) \subseteq y_{1}+\mathscr{K}\left(y_{1}\right) .
$$

Therefore,

$$
\left\{t \in \mathbb{R} \mid t k \in y_{2}+\mathscr{K}\left(y_{2}\right)-\xi\right\} \subseteq\left\{t \in \mathbb{R} \mid t k \in y_{1}+\mathscr{K}\left(y_{1}\right)-\xi\right\} .
$$

This implies the conclusion.

(viii) Assume that $\lambda k \in y+\operatorname{int} \mathscr{K}(y)-\xi$. Then, there exists $\varepsilon>0$ such that

$$
\lambda k+\xi-y-\varepsilon k \in \operatorname{int} \mathscr{K}(y) \subseteq \mathscr{K}(y) .
$$

This implies $T(y) \leq \lambda-\varepsilon<\lambda$.

Conversely, let $y$ such that $T_{\xi}(y)<\lambda$. There exists $t$ such that $T_{\xi}(y)<t<\lambda$ and

$$
t k \in y+\mathscr{K}(y)-\xi .
$$

Taking into account the given property of $k$, we have that

$$
\begin{aligned}
\lambda k & =t k+(\lambda-t) k \\
& \in y+(\mathscr{K}(y)+(\lambda-t) k)-\xi \\
& \subseteq y+\operatorname{int} \mathscr{K}(y)-\xi .
\end{aligned}
$$

Finally, we provide characterizations of nondominated and weak nondominated points to sets via the scalarization function $T_{\xi}$. They play an important role to investigate relationships between a vector optimization problem with a variable domination structure and its corresponding scalarized problem.

Proposition 3.1. (cf. [6, Theorem 5.11]). Let $A \subset Y, \bar{y} \in A$ and the domination structure $\mathscr{K}: Y \rightrightarrows Y$ satisfy $(C 1),(C 2)$.

(i) $\bar{y}$ is a nondominated point of $A$ w.r.t. $\mathscr{K}$ if and only if $\bar{y}$ is a unique minimal solution of $\min _{y \in A} T_{\bar{y}}(y)$, i.e.

$$
T_{\bar{y}}(y)>0 \text { for every } y \in A \backslash\{\bar{y}\} .
$$

(ii) Assume that (C2') holds. Then, $\bar{y}$ is a weakly nondominated point of $A$ w.r.t. $\mathscr{K}$ if and only if $\bar{y}$ is a minimal solution of $\min _{y \in A} T_{\bar{y}}(y)$, i.e.

$$
T_{\bar{y}}(y) \geq 0 \text { for every } y \in A \text {. }
$$

Although [6, Theorem 5.11] was formulated for domination structures with convex cone values, its proof is still valid for the setting under consideration. Therefore, the proof is omitted. 


\section{DUALITY IN VECTOR OPTIMIZATION WITH DOMINATION STRUCTURES}

In this section, let $X, Y$ be two topological vector spaces, $\Omega$ be a nonempty and convex subset of $X$, and $D$ be a proper, pointed and convex cone in $Y$. Let $f, g: X \rightarrow Y$ be vector-valued functions, $\mathscr{K}: Y \rightrightarrows Y$ be a domination structure satisfying $(\mathrm{C} 1)$. Consider a vector optimization problem with a domination structure described by

$$
\mathscr{K}-\text { minimize } f(x) \text { subject to } x \in \mathscr{S},
$$

where the feasible set $\mathscr{S} \subseteq X$ is given by

$$
\mathscr{S}:=\{x \in X: x \in \Omega \text { and } g(x) \in-D\},
$$

and the $\mathscr{K}$-minimality is understood as follows.

Definition 4.1. Consider problem (P). We say that:

(i) $\bar{x}$ is a nondominated solution of problem (P) w.r.t. $\mathscr{K}$ if $f(\bar{x}) \in \mathrm{ND}(f(\mathscr{S}), \mathscr{K})$.

(ii) Assume that int $\mathscr{K}(y) \neq \emptyset$ for all $y \in Y$. $\bar{x}$ is a weakly nondominated solution of problem (P) w.r.t. $\mathscr{K}$ if $f(\bar{x}) \in \mathrm{WND}(f(\mathscr{S}), \mathscr{K})$.

This section is devoted to duality assertions for both nondominated and weakly nondominated solutions to problem $(\mathrm{P})$.

4.1. Duality for weakly nondominated solutions. In this subsection, we always suppose that the following standing assumptions are fulfilled:

The standing assumptions. Let $\mathscr{K}: Y \rightrightarrows Y$ be a domination structure satisfying conditions $(C 1),(C 2)$ and $(C 3), f: X \rightarrow Y$ be $\mathscr{K}$-convex on $\Omega$, and $g: \Omega \rightarrow Y$ be a $D$-convex function.

As usual, we denote the topological dual cone to $D$ by $D^{*}$.

Let $\xi \in Y$ and let $T_{\xi}$ be the nonlinear scalarization function defined by (3.1). We consider the following scalarized problem corresponding to problem $(\mathrm{P})$ :

$$
\text { Minimize } \quad T_{\xi} \circ f(x) \quad \text { subject to } x \in \mathscr{S} \text {. }
$$

The Lagrange function $L: \Omega \times D^{*} \rightarrow \overline{\mathbb{R}}$ associated to problem (SP) is defined by

$$
L\left(x, u^{*}\right):=T_{\xi} \circ f(x)+u^{*} \circ g(x) .
$$

Obviously, if $x \notin \mathscr{S}$, then $g(x) \notin-D$ and thus there exists $u^{*} \in D^{*}$ such that $u^{*} \circ g(x)>0$. Therefore, we have

$$
\forall x \notin \mathscr{S}, \quad \sup _{u \in D^{*}} L\left(x, u^{*}\right)=+\infty .
$$

In addition, if $x \in \mathscr{S}$ then $g(x) \in-D$ and $u^{*} \circ g(x) \leq 0$ for all $u^{*} \in D^{*}$. We have

$$
\forall x \in \mathscr{S}, \quad T_{\xi} \circ f(x)=\sup _{u^{*} \in D^{*}} L\left(x, u^{*}\right) .
$$

Taking into account (4.2) and (4.3), we get

$$
\inf _{x \in \mathscr{S}} T_{\xi} \circ f(x)=\inf _{x \in \Omega} \sup _{u^{*} \in D^{*}} L\left(x, u^{*}\right),
$$


i.e., the scalarized problem (SP) is an inf-sup problem. Therefore (see [10, Satz 5.19]), it has the dual problem of form:

$$
\sup _{u^{*} \in D^{*}} \inf _{x \in \Omega} L\left(x, u^{*}\right)
$$

equivalently,

$$
\sup _{u^{*} \in D^{*}} G\left(u^{*}\right) \text { with } G\left(u^{*}\right):=\inf _{x \in \Omega} L\left(x, u^{*}\right) .
$$

We define a dual problem of problem $(\mathrm{P})$ by

$$
\operatorname{Max}\left(\mathscr{S}_{D}, \mathscr{K}\right)
$$

where

$$
\mathscr{S}_{D}:=\left\{y \in Y: \exists u^{*} \in D^{*}, \forall x \in \Omega, T_{y} \circ f(x)+u^{*} \circ g(x) \geq 0\right\} .
$$

We always assume that $\mathscr{S}_{D} \neq \emptyset$. The next proposition provides an important property of elements in this set.

Proposition 4.1. Let the standing assumptions be satisfied. For every $\xi \in \mathscr{S}_{D}$ it holds that

$$
\forall y \in f(\mathscr{S}): \quad T_{\xi}(y) \geq 0=T_{\xi}(\xi) .
$$

Proof. Take $\xi \in \mathscr{S}_{D}$ arbitrarily. By the definition of $\mathscr{S}_{D}$, there exists $u^{*} \in D^{*}$ such that

$$
T_{\xi} \circ f(x)+u^{*} \circ g(x) \geq 0 \text { for all } x \in \Omega .
$$

Since $g(x) \in-D$ for every $x \in \mathscr{S}, u^{*} \circ g(x) \leq 0$ for every $x \in \mathscr{S}$, which implies the assertion (4.5).

Theorem 4.1. (Weak duality) Let the standing assumptions be satisfied. Then, the following assertion holds true:

$$
\mathscr{S}_{D} \cap\left[\cup_{x \in \mathscr{S}}(f(x)+\operatorname{int} \mathscr{K}(f(x))]=\emptyset .\right.
$$

Moreover, if $\bar{y} \in \mathscr{S}_{D} \cap f(\mathscr{S})$, i.e. $\bar{y}=f(\bar{x})$ for some $\bar{x} \in \mathscr{S}$, then $\bar{x}$ is a weakly nondominated solution of the primal problem (P) w.r.t $\mathscr{K}$ and $\bar{y}$ is a weakly maximal solution of the dual problem (D) w.r.t. the variable structure $\mathscr{K}$.

Proof. Arguing by contradiction, we assume that (4.6) does not hold. Then, there exist $\xi \in \mathscr{S}_{D}$ and $\bar{x} \in \mathscr{S}$ such that $\xi \in f(\bar{x})+\operatorname{int} \mathscr{K}(f(\bar{x}))$. The later ensures the existence of $\varepsilon>0$ such that

$$
-\varepsilon k \in f(\bar{x})+\mathscr{K}(f(\bar{x}))-\xi,
$$

which implies that $T_{\xi}(f(\bar{x})) \leq-\varepsilon<0$. This contradicts the inequality $T_{\xi}(f(\bar{x})) \geq 0$ in Proposition 2 and thus verifies (4.6). Assume in addition that there is $\bar{x} \in S$ satisfying $\bar{y}=f(\bar{x}) \in$ $\mathscr{S}_{D} \cap f(S)$. We get from (4.6) that

$$
\forall y \in f(S) \backslash\{f(\bar{x})\}, \exists x \in S: y=f(x) .
$$

By (4.1) we have

$$
\bar{y} \notin y+\operatorname{int} \mathscr{K}(y)
$$

This means that $\bar{x}$ is a weakly nondominated solution of problem (P). Assume that $\bar{y}$ is not a weakly nondominated solution of problem (D). Then, there is $y \in \mathscr{S}_{D} \backslash\{\bar{y}\}$ such that

$$
y \in \bar{y}+\text { int } \mathscr{K}(\bar{y}) \Longleftrightarrow \bar{y} \in y-\operatorname{int} \mathscr{K}(\bar{y}) .
$$


Then, $T_{y}(\bar{y})<0$. For every $u^{*} \in D^{*}$, we have

$$
\left(T_{y} \circ f+u^{*} g\right)(\bar{x})<0 .
$$

The above assertion contradicts $y \in \mathscr{S}_{D}$. The proof is complete.

To derive a strong duality theorem, we need the lower semicontinuity of the function $T_{\xi} \circ f$. Below is a sufficient condition.

Proposition 4.2. [1] Suppose that $f$ is continuous over $\Omega \subset X, \xi \in Y$ and the restricted map $\left.\mathscr{K}\right|_{\Omega}$ has a closed graph in the sense that

$\left(C_{5}\right)$ For every sequence $\left(y_{n}, t_{n}\right)$ such that $y_{n} \in f(\Omega)$ and $t_{n} \in \mathscr{K}\left(y_{n}\right)$ for all $n \in \mathbb{N}$ and $\left(y_{n}, t_{n}\right) \rightarrow\left(y^{*}, t^{*}\right)$ as $n \rightarrow+\infty$ then $y^{*} \in f(\Omega)$ and $t^{*} \in \mathscr{K}\left(y^{*}\right)$.

Then, $T_{\xi} \circ f$ is lower semicontinuous on $\Omega$.

Theorem 4.2. (Strong duality) Let the standing assumptions hold. If $\bar{x} \in \Omega$ is a weakly nondominated solution w.r.t. $\mathscr{K}$ of problem $(\mathrm{P})$, then $\bar{y}:=f(\bar{x})$ is a weakly maximal point of $\mathscr{S}_{D}$ w.r.t. $\mathscr{K}$ provided that one of the following assumptions is satisfied:

(i) (Slater constraint qualification) $X, Y$ are Hausdorff locally convex spaces and there is $x^{\prime} \in \operatorname{dom} f \cap \Omega$ such that $g\left(x^{\prime}\right) \in \operatorname{int} D$.

(ii) $X, Y$ are Fréchet spaces, $\Omega$ is closed and the domination structure $\mathscr{K}$ satisfies $\left(C_{5}\right)$, epig is closed and $0 \in \operatorname{sqri}\left(g\left(\operatorname{dom} T_{\bar{y}} \circ f \cap \Omega \cap \operatorname{dom} g\right)+D\right)$.

(iii) $X, Y$ are finite dimensional spaces. In addition, it is assumed that the following two conditions hold true

$$
\operatorname{dim}\left(\operatorname{lin}\left(g\left(\operatorname{dom} T_{\bar{y}} \circ f \cap \Omega \cap \operatorname{dom} g\right)+D\right)\right)<+\infty
$$

and

$$
0 \in \operatorname{ri}\left(g\left(\operatorname{dom} T_{\bar{y}} \circ f \cap \Omega \cap \operatorname{dom} g\right)+D\right) .
$$

Proof. Since $\bar{y}=f(\bar{x})$ is a weakly nondominated point of $f(\mathscr{S})$ w.r.t. $\mathscr{K}$, we obtain

$$
\forall y \in f(\mathscr{S}), \quad T_{\bar{y}}(y) \geq 0
$$

by Proposition 3.1 .

Applying [3, Theorem 3.2.9], one of the three assumptions (i)-(iii) ensures the existence of $\bar{u}^{*} \in D^{*}$ such that

$$
\inf _{x \in \Omega} \sup _{u^{*} \in D^{*}} L\left(x, u^{*}\right)=\sup _{u^{*} \in D^{*}} \inf _{x \in \Omega} L\left(x, u^{*}\right)=\inf _{x \in \Omega} \quad\left(T_{\bar{y}} \circ f+\bar{u}^{*} \circ g\right)(x) .
$$

Furthermore, by (4.4) we have

$$
\inf _{x \in \Omega} \sup _{u^{*} \in D^{*}} L\left(x, u^{*}\right)=\inf _{x \in \mathscr{S}}\left(T_{\bar{y}} \circ f\right)(x) .
$$

Taking into account (4.5) and (4.7), the following assertion holds true for all $\hat{x} \in \Omega$ :

$$
\left(T_{\bar{y}} \circ f+\bar{u}^{*} \circ g\right)(\hat{x}) \geq \inf _{x \in \mathscr{S}}\left(T_{\bar{y}} \circ f\right)(x)=\left(T_{\bar{y}} \circ f\right)(\bar{x})=0,
$$

clearly verifying that $\bar{y} \in \mathscr{S}_{D}$. 
Now, we prove that $\bar{y}$ is a weakly maximal element of the set $\mathscr{S}_{D}$ w.r.t. $\mathscr{K}$. Arguing by contradiction, we assume that $\exists y \in \mathscr{S}_{D} \backslash\{\bar{y}\}$ satisfying $\bar{y} \in y-\operatorname{int} \mathscr{K}(\bar{y})$. Then, $T_{y}(\bar{y})<0$. For every $u^{*} \in D^{*}$, we have

$$
\left(T_{y} \circ f+u^{*} \circ g\right)(\bar{x})<0
$$

which contradicts $y \in \mathscr{S}_{D}$.

Remark 4.1. Eichfelder [7, Section 8.3] used linear scalarization to investigate duality assertions for minimal solutions of convex problem $(\mathrm{P})$. For nondominated solutions of problem (P) equipped with Bishop-Phelps cones, a nonlinear scalarization method is used to deal with problem $(\mathrm{P})$ with linear cost function.

\section{Example 4.1. Duality for multiobjective location problems with variable domination struc- tures}

In this example, we will construct duality problems by using our presented results for multiobjective location problems:

$$
\mathscr{K}-\operatorname{Min} f(x), x \in \mathbb{R}^{2}
$$

where $f: \mathbb{R}^{2} \rightarrow \mathbb{R}^{p}$ is determined by

$$
f(x):=\left(\begin{array}{c}
\left\|x-a^{1}\right\| \\
\cdots \\
\left\|x-a^{p}\right\|
\end{array}\right)
$$

$\|\cdot\|$ is a norm in $\mathbb{R}^{2}$ and $a^{i}, i=1, \ldots, p$, and the variable domination $\mathscr{K}: \mathbb{R}^{p} \rightrightarrows \mathbb{R}^{p}$ defined by:

$$
\mathscr{K}(y):= \begin{cases}\mathbb{R}_{+}^{p} & \text { if } y \neq\left(\left\|a^{1}\right\|, \ldots,\left\|a^{p}\right\|\right)^{T}, \\ \left\{y \in \mathbb{R}^{p} \mid y=\alpha(1,1, \ldots, 1)^{T}, \alpha \geq 0\right\} & \text { otherwise. }\end{cases}
$$

Observe that duality results for this problem equipped with a domination structure given by a fixed cone are investigated in [9], where the authors dealt with general approximation problems.

With the variable domination structure given above, it is clear that we can choose $k=$ $(1, \ldots, 1)$ such that it satisfies conditions $(C 1)$ and $(C 2)$. Now, we follow the scheme given in this section to derive dual problem for our problem. In this setting, $g \equiv 0$ and $\mathscr{S}=\Omega=\mathbb{R}^{p}$. Therefore, the Lagrange function is determined as:

$$
L(x)=T_{\xi} f(x) .
$$

Thus, the dual problem is

$$
\mathscr{K}-\operatorname{Max}\left(\mathscr{S}_{D}, \mathscr{K}\right)
$$

where

$$
\mathscr{S}_{D}=\left\{y \in \mathbb{R}^{p}: \forall x \in \mathbb{R}^{2}, T_{y} f(x) \geq 0\right\} .
$$

Taking into account (3.1), for all $x \in \mathbb{R}^{2}$ and $t<0$ the following assertion is fulfilled for all $y \in \mathscr{S}:$

$$
y \notin f(x)+\mathscr{K}(f(x))-t k .
$$

Because of the definition of the domination structure $\mathscr{K}$, it holds that

$$
\mathscr{K}(f(x))-t k=\mathscr{K}(f(x)), \forall x \in \mathbb{R}^{2} .
$$


This implies that for all $y \in \mathscr{S}_{D}: y \notin \bigcup_{x \in \mathbb{R}^{2}}(f(x)+\mathscr{K}(f(x))$. In addition, based on definition of $\mathscr{K}$ we set

$$
\mathscr{S}_{1}:=\bigcup_{x \neq 0}\left(f(x)+\mathbb{R}_{+}^{p}\right)
$$

and

$$
\mathscr{S}_{2}:=\left\{y \in \mathbb{R}^{p} \mid y=\left(\left\|a^{1}\right\|, \ldots,\left\|a^{p}\right\|\right)^{T}+\alpha(1, \ldots, 1)^{T}, \alpha \geq 0\right\} .
$$

Therefore, $\mathscr{S}_{D}=\mathbb{R}^{p} \backslash\left(\mathscr{S}_{1} \cup \mathscr{S}_{2}\right)$ and thus the weak duality result is obvious.

Remark 4.2. A natural question possibly arises is that whether the relation $\mathscr{S}_{2} \subseteq \mathscr{S}_{1}$ always holds true. It also means that there is $m^{*} \in \mathbb{R}^{2} \backslash\{0\}$ such that $\left(\left\|a^{1}\right\|,\left\|a^{2}\right\|, \ldots,\left\|a^{p}\right\|\right)^{T} \geq_{\mathbb{R}_{+}^{p}}$ $\left(\left\|a^{1}-m^{*}\right\|,\left\|a^{2}-m^{*}\right\|, \ldots,\left\|a^{p}-m^{*}\right\|\right)^{T}=f\left(m^{*}\right)$. This could be happened for instance $\|\cdot\| \equiv$ $\|\cdot\|_{2}, a^{i} \geq_{\mathbb{R}_{+}^{2}} 0, i=1, \ldots, p$ and we take $m_{1}^{*}:=\min \left\{a_{1}^{i}\right\}$ and $m_{2}^{*}:=\min \left\{a_{2}^{i}\right\}, i=1, \ldots, p$. However, in general cases we can not always show that $\mathscr{S}_{2} \subseteq \mathscr{S}_{1}$.

4.2. Duality for nondominated solutions. In this part, we investigate duality assertions for the problem $(\mathrm{P})$ where nondominated solutions are defined in the sense of Definition 4.1 and the domination structure $\mathscr{K}: Y \rightrightarrows Y$ satisfies property $(C 1)$. We assume that the set $\mathfrak{S}$ of all strictly $\mathscr{K}$-monotone functions $s: Y \rightarrow \mathbb{R}$ is nonempty. This will be used to derive a strong duality result and we also prove the function $\bar{T}_{\xi}$ (given by (4.13)) belongs to this set under appropriate assumptions. Let $\Omega^{*}$ be a nonempty set and consider a generalized Lagrange function $L: \Omega \times$ $\Omega^{*} \rightarrow Y \cup\{+\infty\}$ such that

$$
\forall s \in \mathfrak{S}, \forall g(x) \in-D: \quad \sup _{y \in \Omega^{*}} s(L(x, y))=s(f(x)) \quad \text { if } g(x) \in-D .
$$

The existence and well-definedness of the generalized Lagrange function $L$ in this part are supposed to be fulfilled. We define a dual problem as follows:

$$
\operatorname{Max}\left(\overline{\mathscr{S}_{D}}, \mathscr{K}\right)
$$

where

$$
\overline{\mathscr{S}_{D}}=\left\{h \in Y \mid \exists s \in \mathfrak{S}, \exists y \in \Omega^{*}: s(h)=\inf \{s(L(x, y)) \mid x \in \Omega\}>-\infty\right\} .
$$

For elements $h \in \overline{\mathscr{S}_{D}}$, we say that $s$ is the corresponding function to $h$ if the conditions in the definition of $\overline{\mathscr{S}_{D}}$ are fulfilled for $h$ and $s$ ( $s$ corresponds to $h$ according to the definition of $\overline{\mathscr{S}_{D}}$ ).

Theorem 4.3. (Weak duality) Consider problems $(\mathrm{P})$ and $(\overline{D P})$. Let $\mathscr{K}: Y \rightrightarrows Y$ satisfy condition (C1). Then, we have

$$
\overline{\mathscr{S}_{D}} \cap \cup_{u \in \mathscr{S}}(f(u)+\mathscr{K}(f(u)) \backslash\{0\})=\emptyset .
$$

Proof. Suppose by contradiction that there is $u_{0} \in \mathscr{S}$ and $z_{0} \in \overline{\mathscr{S}_{D}}$ such that

$$
z_{0} \in f\left(u_{0}\right)+\mathscr{K}\left(f\left(u_{0}\right)\right) \backslash\{0\} .
$$

Since $z_{0} \in \overline{\mathscr{S}_{D}}$, we take $s_{0}$ corresponding to $z_{0}$. We get that $f\left(\underline{\left.u_{0}\right)} \prec \mathscr{K} z_{0}\right.$ and thus $s_{0}\left(f\left(u_{0}\right)\right)<$ $s_{0}\left(z_{0}\right)$, where $s_{0} \in \mathfrak{S}$ corresponding to $z_{0} \in \overline{\mathscr{S}_{D}}$. Since $z_{0} \in \overline{\mathscr{S}_{D}}$, there is $y_{0} \in \Omega^{*}$ such that 
$s_{0}\left(z_{0}\right)=\inf _{x \in \Omega} s_{0}\left(L\left(x, y_{0}\right)\right)$. On the other hand, since $\sup _{y \in \Omega^{*}} s_{0}(L(x, y))=s_{0}(f(x)), y_{0} \in \Omega^{*}$ we have that

$$
s_{0}\left(z_{0}\right)=\inf _{x \in \Omega} s_{0}\left(L\left(x, y_{0}\right)\right) \leq s_{0}\left(L\left(u_{0}, y_{0}\right)\right) \leq \sup _{y \in \Omega^{*}} s_{0}\left(L\left(u_{0}, y\right)\right)=s_{0}\left(f\left(u_{0}\right)\right) .
$$

This implies $s_{0}\left(z_{0}\right) \leq s_{0}\left(f\left(u_{0}\right)\right)$, a contradiction.

Remark 4.3. Theorem 4.3 is still valid if we replace the condition (4.10) by a weaker assumption, namely $s(L(x, y)) \leq s(f(x))$ provided that $g(x) \in-D$. This observation goes back to the process of the proof given above.

Definition 4.2. $\bar{x}$ is called a proper solution of problem (P) if there is $\bar{s} \in \mathfrak{S}$ such that $\bar{s}(f(\bar{x}))=$ $\inf \{\bar{s}(f(x)) \mid x \in \mathscr{S}\}>-\infty$.

Observe that every proper solution of problem $(\mathrm{P})$ is a nondominated solution. Indeed, suppose that $\bar{x}$ is a proper solution and $\bar{s}$ is a corresponding function as in Definition 5 . Then, for all $y \in f(\mathscr{S}) \backslash\{0\}$, we have $y \npreceq \mathscr{K} \bar{y}$; otherwise, assume that $y \preceq \mathscr{K} \bar{y}$, then $\bar{s}(y)<\bar{s}(\bar{y})$ since $s \in \mathfrak{S}$ and thus this contradicts the definition of $\bar{s}$.

In the following, we derive a result of strong duality, where proper solutions of $(\mathrm{P})$ are involved. This also means that it holds true for a subset of the set of nondominated solutions.

Theorem 4.4. (Strong duality) Consider problems $(\mathrm{P})$ and $(\overline{D P})$ and assume that $\mathscr{K}: Y \rightrightarrows Y$ satisfies condition $(C 1)$. If $\bar{x} \in \mathscr{S}$ is a proper solution of problem $(\mathrm{P})$ w.r.t. $\mathscr{K}$ then $\bar{y}=f(\bar{x})$ is a maximal solution of problem $(\overline{D P})$ w.r.t. $\mathscr{K}$.

Proof. Let $\bar{x}$ be a proper solution of (P). Then, there is $\bar{s} \in \mathfrak{S}$ such that we can choose the Lagrange function for $\bar{y} \in \Omega^{*}$ as $\bar{s}(L(x, \bar{y}))=\bar{s}(f(x))$ if $g(x) \in-D$ and $\bar{s}(L(x, \bar{y}))=+\infty$ otherwise. Therefore,

$$
\bar{s}(f(\bar{x}))=\inf _{x \in \mathscr{S}} \bar{s}(L(x, \bar{y}))=\inf \left\{\inf _{x \in \mathscr{S}} s(L(x, \bar{y})), \inf _{x \in \Omega \backslash \mathscr{S}} s(L(x, \bar{y}))\right\}=\inf _{x \in \Omega} s(L(x, \bar{y})) .
$$

This means that $f(\bar{x}) \in \overline{\mathscr{S}_{D}}$. By Theorem 4.3, there is no $z \in \overline{\mathscr{S}_{D}}$ such that $z \in f(\bar{x})+\mathscr{K}(f(\bar{x}))$. This implies that $\bar{x}$ is maximal solution of $(\overline{D P})$ w.r.t. $\mathscr{K}$.

At this time, a natural question arises whether it is possible to utilize the function $T_{\xi}$ defined by (3.2) as a suitable scalarized function in the set $\mathfrak{S}$ including strictly $\mathscr{K}$-monotone functions. We will show with a mild change in definition of the function $T_{\xi}$ the strictly $\mathscr{K}$-monotone property will be fulfilled.

For the next result, we need the following assumption.

Assumption 4.1. $\mathscr{K}: Y \rightrightarrows Y$ be a domination structure satisfy $(C 1)$ and there is a set-valued map $\mathscr{B}: Y \rightrightarrows Y$ satisfying $(\mathrm{C} 1),\left(\mathrm{C} 2^{\prime}\right),(\mathrm{C} 4)$ and

$$
\forall y \in Y, d \in \mathscr{K}(y): \mathscr{K}(y) \backslash\{0\}+\mathscr{B}(y+d) \subseteq \operatorname{int} \mathscr{B}(y) .
$$

Now, for a given element $\xi \in Y$, we introduce a function $\bar{T}_{\xi}: Y \rightrightarrows \overline{\mathbb{R}}$ as follows:

$$
\bar{T}_{\xi}(y):=\inf \{t \in \mathbb{R} \mid t e \in y+\mathscr{B}(y)-\xi\}
$$


Obviously, all properties of the function $T_{\xi}$ given in Theorem 3.1 hold true for the function $\bar{T}_{\xi}$, where the domination structure $\mathscr{K}$ is replaced by $\mathscr{B}$. Furthermore, $\bar{T}_{\xi}$ is strictly $\mathscr{K}$-monotone.

Proposition 4.3. Let Assumption 4.1 hold true and $e \in Y \backslash\{0\}$ such that for all $y \in Y, \mathscr{B}(y)+$ $(0,+\infty) e \subseteq$ int $\mathscr{B}(y)$. Then, the function $\bar{T}_{\xi}$ given by (4.13) is strictly $\mathscr{K}$-monotone.

Proof. Assume that $y_{1} \preceq \mathscr{K} y_{2}$, i.e., $y_{2} \in y_{1}+\mathscr{K}\left(y_{1}\right) \backslash\{0\}$. We will prove that $\bar{T}_{\xi}\left(y_{1}\right)<\bar{T}_{\xi}\left(y_{2}\right)$. By definition of function $\bar{T}_{\xi}$, we have that

$$
\bar{T}_{\xi}\left(y_{2}\right) e \in y_{2}+\mathscr{B}\left(y_{2}\right)-\xi \subseteq y_{1}+\mathscr{K}\left(y_{1}\right)+\mathscr{B}\left(y_{1}+\mathscr{K}\left(y_{1}\right)\right)-\xi .
$$

Taking into account (4.12), it holds that

$$
\bar{T}_{\xi}\left(y_{2}\right) e \in y_{1}+\operatorname{int} \mathscr{B}\left(y_{1}\right)-\xi .
$$

Taking into account Theorem 3.1(v), we obtain that $\bar{T}_{\xi}\left(y_{1}\right)<\bar{T}_{\xi}\left(y_{2}\right)$.

Remark 4.4. Proposition 4.3 shows that it is possible to get duality results for problem (P) by using the function $\bar{T}_{\xi}$ under the Assumption 4.1. The procedure used in this part is also studied in [9, Section 3.7] for vector optimization problems with fixed ordering cones.

\section{CONCLUSION}

The present paper deals with duality for vector optimization problems with variable domination structures. Weak duality and strong duality results are given for both weakly nondominated and nondominated solutions. These results are considered as extensions of many works on duality in the literature, where the domination structures are given by fixed cones. In particular, we do not need any linearity assumption on the objective function as in [6, Section 8.3] and the variable domination map is not necessary cone-valued. This approach implies many new results for special cases when we deal with problems where the solution concept is defined by a proper, closed, pointed set. It would be also of interest to derive a inverse strong duality statement (see [9] for the case of fixed ordering cones). In addition, some duality assertions in linear optimization, approximation theory and their applications can be extended using our approaches.

\section{REFERENCES}

[1] T. Q. Bao, G. Eichfelder, B. Soleimani, C. Tammer, Ekeland's variational principle for vector optimization with variable ordering structure, J. Convex Anal. 24 (2017), 393-415.

[2] J. Y. Bello Cruz, G. Bouza Allende, A steepest descent-like method for variable order vector optimization problems, J. Optim. Theory Appl. 162 (2014), 371-391.

[3] R.I. Bots, S.M. Grad, G. Wanka, Duality in Vector Optimization, Springer-Verlag, Berlin, 2009.

[4] A. Doagooei, T.T. Le, C. Tammer, Convexity in the framework of variable domination structures and applications in optimization, J. Nonlinear Convex Anal. in press.

[5] G. Eichfelder, T.X.D. Ha, Optimality conditions for vector optimization problems with variable ordering structures, Optimization, 62 (2013), 597-627.

[6] G. Eichfelder, Variable Ordering Structures in Vector Optimization, Springer, Heidelberg, 2014.

[7] G. Eichfelder, Vector optimization in medical engineering, In: Mathematics without boundaries, 181-215, Springer, New York, 2014.

[8] C. Gerth, P. Weidner, Nonconvex separation theorems and some applications in vector optimization, J. Optim. Theory Appl. 67 (1990), 297-320. 
[9] A. Göpfert, H. Riahi, C. Tammer, C. Zălinescu, Variational Methods in Partially Irdered Spaces, CMS Books in Mathematics/Ouvrages de Mathématiques de la SMC, 17. Springer-Verlag, New York, 2003.

[10] A. Göpfert, T. Riedrichm C. Tammer, Angewandte Funktionalanalysis, Teubner+Vieweg, 2009.

[11] T.T. Le, Multiobjective approaches based on variable ordering structures for intensity problems in radiotherapy treatment, Investigación Oper. 39 (2018), 426-448.

[12] D.T. Luc, Theory of vector optimization, volume 319 of Lecture Notes in Economics and Mathematical Systems, Springer-Verlag, Berlin, 1989.

[13] V.A. Tuan, C. Tammer, C. Zălinescu, The Lipschitzianity of convex vector and set-valued functions, TOP 24 (2016), 273-299. 